\title{
Condition Monitoring System of Package Box Based on PHM
}

\author{
Wang Qian \\ Department of Aviation Ammunition \\ Air Force Logistics College \\ Xuzhou, China \\ LI Zhiqiang \\ Department of Aviation Ammunition \\ Air Force Logistics College \\ Xuzhou, China
}

\author{
Rong Penghui \\ Department of Aviation Ammunition \\ Air Force Logistics College \\ Xuzhou, China \\ LI Genyan \\ Department of Aviation Ammunition \\ Air Force Logistics College \\ Xuzhou, China
}

\begin{abstract}
To estimate the health status of equipment inside the package box, monitor the vibration and shock during transporting or in store, a condition monitoring system is established based on PHM and sophisticated sensor technology. Through storage life analysis of a missile, the benefit of package box applied PHM is discussed for equipment support. By analyzing environmental factors, monitoring parameters are determined, requirements are put forward according to the size and cost of sensor systems, and the monitoring model is established. By carrying out a survey, sensor systems available are determined, and expectation is expressed for sensor systems used inside the equipment in the future.
\end{abstract}

Keywords- health status; package box; condition monitoring system; РHM; sensor system

\section{INTRODUCTION}

Expensive and sophisticated equipments such as missiles and satellites, are usually settled in special package boxes when transporting or in store. Although there are pads in the boxes, these equipments still endure great vibration and shock in transportation. Temperature, humidity, acidity and alkalinity are challenges for them when in store. The shortages of support modes in opencase inspection and periodic test become obvious. If the advantages of prognostics and health management can be used, the visibility of package box will be improved, and the support mode will be optimized.

\section{BRIEF INTRODUCTION OF PHM}

\section{A. Conception of PHM}

With fault diagnosis technique becoming more intelligent and "Economic Affordability" put on schedule, the concept of Prognostic and Health Management is put forward on the base of condition based maintenance and autonomic logistics. PHM refers to use less sensor systems, and take advantage of intelligent algorithms to estimate the health status of the system, so that the fault can be detected before it happens[1]. A series of actions can be taken according to the prediction. PHM is put forward in the plan of Joint Strike Flight in U.S. But its full development is displayed on F-35[2]: the application of PHM has changed the maintenance theory. Fault detection is available in every important system of aircraft, and the fault can be diagnosed and located precisely. The remaining useful life of critical parts can be predicted. The number of intermediate test equipments and development and verification equipments has decreased. The repair-delayed time becomes short, support efficiency has been improved, the cost of maintenance has decreased, and the training has been simplified. From the cost-benefit analysis of PHM applied to traditional legacy, we can see great value in the research of PHM.

\section{B. National and international research on PHM}

U.S. Military puts forward the concept of PHM initially. It requires being able to predict the fault of the system, and helping to complete health management. Patrick and Chin Sun explained the theory of PHM, and predicted the benefit it may bring for maintenance and logistics support[3,4]. The application of PHM was carried out on helicopter firstly, and then HUMS system was formed. With the spread of application, U.S. invented a lot of systems, such as Aircraft Condition Monitoring System, Integrated Diagnostics and Prognostics System, Engine Monitoring System and Integrated Condition Assessment System. According to these models, Richard M. analyzed the cost-benefit of CBM based on PHM, and explained the influence it brought to service and support[5]. In America, PHM has been applied to traditional legacies, big dams, nuclear power plants, automotives and bridges.

The research on PHM in domestic starts later relatively, but it develops quickly. Zeng Sheng-kui gave a report on PHM in 2005[6], and then many scientific research institutes and colleges began to make great efforts on PHM: Zhang Bao-zhen introduced the application of PHM abroad in both civil field and military field, predicted the future development tendency of PHM by analyzing its application on F-35[7]. Wu Ming-hui established the frame structure of the integrated avionic system according to 
OSA-CBM, and studied the application of PHM in this field[8]. Liu Jin-ning abstracted the messages of PHM system, and inductived the relationship between information entities, and established a normalized model based on XML language[9]. So far, the researches and experiments about PHM mainly concentrate on military equipments, such as military aircrafts, radars, engines, bearing and armored vehicles. Wang Qing-hai studied the application of PHM on military communication system for command. Wang Han-zhong established a radar maintenance support system based on PHM, and it was useful to detect faults in radar systems and predict the remaining useful life[10]. Liu Peng-peng discussed the aircraft maintenance decision theory when PHM applied[11].

\section{PACKAGE BOX LIFE-CYCLE MANAGEMENT BASED ON PHM}

The package box is mainly in two conditions, in store or in transportation, depended on the equipment in it. Figure 1 is the sectional graph of a certain missile in storage life in service. It can be inferred that the missile needs test many times when in store, in transportation or ready to use. However, testing is some kind of consumption. If PHM is applied to the package box when designed and manufactured, it may be possible to monitor vibration, shock and acceleration in transportation, Loading and Unloading. By analyzing these data, the condition of the equipment inside can be inferred, and the potential faults can be detected. Equipment technical support technicians can take actions accordingly. Condition based maintenance applying PHM replaces periodic test, relieves the support burden, and saves a plenty of human resource and material resource. And it improves the quality of package box, ensures the reliability of equipment inside.

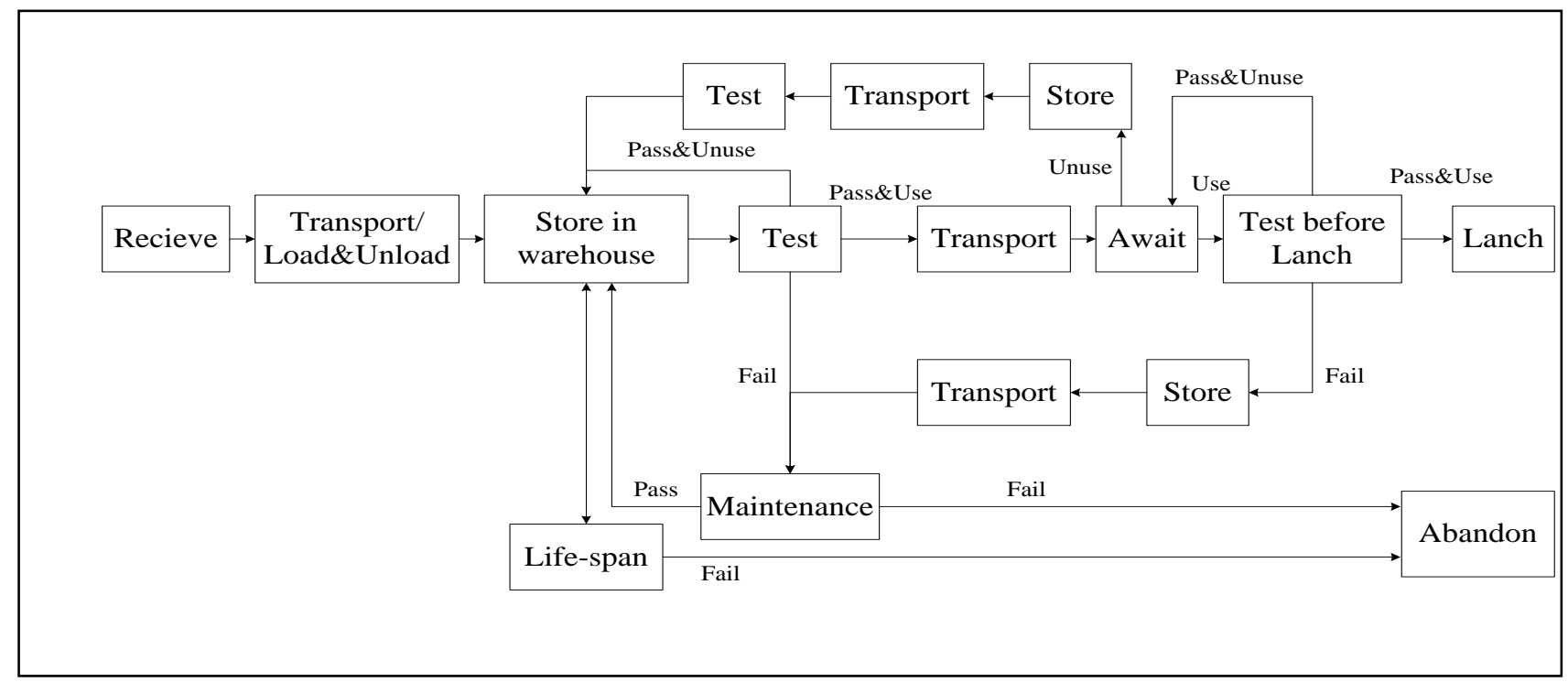

Figure1. Sectional graph of a certain missile in storage life

\section{ESTABLISH THE CONDITION MONITORING SYSTEM OF PACKAGE BOX}

The parameters need to be monitored are determined according to the environment and the purpose of package box. With the help of sensor systems to collect data and microprocessor to analyze, the condition of the equipment can be predicted easily.

\section{A. monitoring parameters}

To establish the condition monitoring system of package box, sensor systems must be used to collect and analyze these environmental parameters, such as temperature, humility, vibration, acceleration and atmospheric pressure.

1)Temperature: The temperature inside the package box should remain beyond thirty centigrade, twenty is better. Too high, too low, or change acutely, the temperature will affect the equipment. If the temperature is too high, corrosion of metal parts of the equipment will be accelerated, aging behavior of rubber, plastics and protective layer will be promoted. If the temperature is too low, the strength of rubber of the equipment will be lowed, the plastics will crack easily, and the protective layer will drop down. If the temperature changes acutely, the transistor of electronic components of the equipment will broke.

2)Humility: The humility needs to stay within a range beyond seventy percent, forty percent is better. If the humility is too low, the leather of the equipment will crack. If the humility is too high, the metal parts will corrode, the package box, paper, cloth and leather will be damp and decay, and the performance of plastics will be low.

3)Vibration: Vibration sensor systems are used to monitor the shock the package box endures. Seismic detector helps to record the vibration around the equipment, such as capturing big shock caused by people or machines. 
Frequent shock will move fasteners inside the equipment from their original positions.

4) Acceleration: To prevent metal parts from being oxidized by the air, the equipment is covered by bag in vacuum or filled with nitrogen. When the pressure changed obviously, sensor system inside the package box will detect it in time. If the bag is in vacuum, there air-leakage happens, the air outside will get into the package box, then the pressure will rise. If the bag is filled with nitrogen, there air-leakage happens, the nitrogen gets out, and then the pressure will lower. The other purpose of package box in vacuum or filled with nitrogen is to prevent mushroom from reproducing.

5) Atmospheric pressure: Acceleration sensor system fixed inside the package box is used to monitor the equilibrium state of the node. It's critical to prevent sliding. Set the mode of sensor system to save power: in regular condition, the acceleration of package box is zero, the node is in a low power consumption mode. Once there is acceleration, the sensor system turns into working mode immediately, collects data and analyzes it. If the data is out of the setting criteria range, it can be inferred that the equipment is unbalanced. Monitoring node turns into working mode immediately, and alarms to call for tackling.

\section{B. Requirements for sensor system}

The sensor system applied to PHM should be chosen according to its cost, size and weight, and the range of measurement parameters. Because the sensor system is limited by its power, transmission mode, onboard memory, we should choose a sensor with battery inside or outside giving the actual condition, and decide whether wireless or wired transmission. The settlement of sensor node inside should not affect the structure and construction of package box, that is to say, minimizing damage to it. And at the same time, the data can be collected precisely and timely. MEMS sensors with small size and low power consumption are suited for the case. As sensors consuming less power and alerting between working mode, idle mode and sleeping mode, a battery can be available for one year or two. If sensors with data readout are used, the system can decide whether to transmit the data by comparing it with the setting range.

\section{Mode of condition monitoring system of package box}

The hardware of the condition monitoring system of package box consists of sensor system, microprocessor, data processing center of the warehouse and relative response equipments. The node can collect the indexes of temperature, humility, vibration inside the package box. If the parameter is within the setting range, it will not be transmitted. If it's out the setting range, it will be transmitted to the microprocessor in wired or wireless method. With the help of expert system or inference algorithm, the data is analyzed comprehensive, and then the result is sent to data processing center. If the problem happens in many package boxes, such as high temperature or high humility, center conditioner will accommodate the temperature or humility to make a better environment.
However, if a sudden shock or high temperature happens in one package box, the microprocessor will call for dealing with its alarming device, and send data to the monitoring center meanwhile.

The display module of microprocessor in the condition monitoring system can show the working condition of every sensor system. If one sensor system is out of work, the display module will recognize it, and the technician in the warehouse can fix or replace it timely, so that there is no dead-angle in the monitoring zone of the package box in the warehouse. The setting range calling for dealing can be set differently at different places, and in different weather conditions. The network structural model inside the package box is described as Figure 2 below. If the package box is made of metal, the wireless method for data transmission inside it is unavailable. Wired method is adopted then.

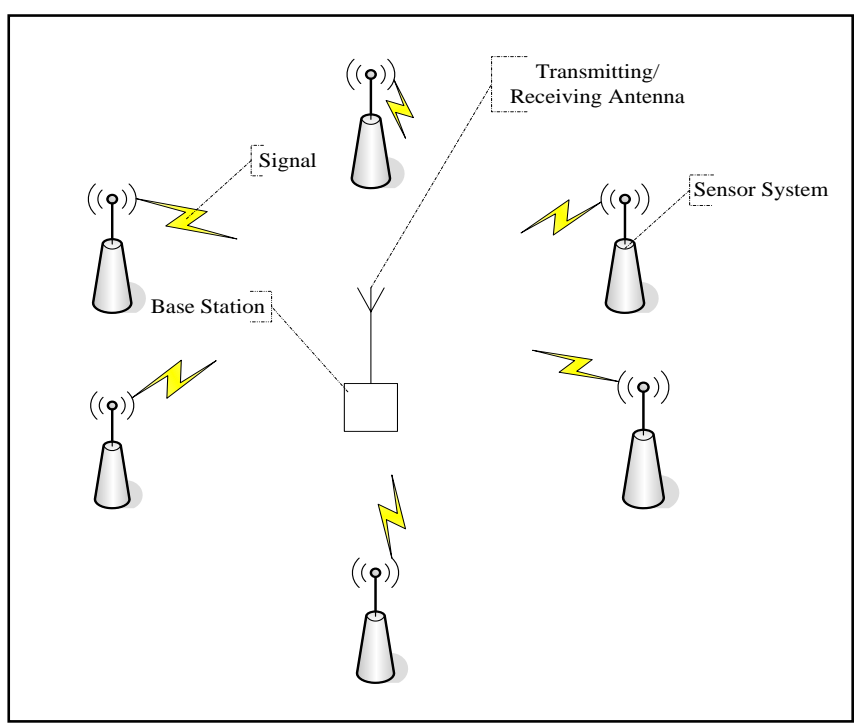

Figure2. Network structural model of sensor systems inside the package box

\section{CHOICE OF SENSOR SYSTEMS}

To determine sensor systems available in condition monitoring system of package box on sale, the performance information of thirty-one sensor systems from twenty-two manufactures is collected. The information includes sensing indexes, power supplement and management, sampling frequency, memory onboard, data transmission mode, availability of signal processing software inside, size, weight and cost. There are many typical sensor systems, such as TMP275 temperature sensor produced by Texas Instruments Company, SHT15 humidity sensor produced by Sensirion Company, SMB380 vibration sensor with data readout produced by BOSCH Company, SCA3000-E01 acceleration sensor produced by VTI Technologies Company, MS5534B atmospheric pressure sensor produced by Intersema Company.

To use sensor system in equipments applied PHM, it must improve in several aspects, and size and weight come at first. An electronic system applied PHM consists of 
components in high density, and smaller and lighter sensor is needed to fix on the circuit board. And power supply on board is a critical limitation as well, especially for sensor system transmitting data in wireless mode. If power runs out, the battery must be charged or replaced quickly. In PHM application, non-battery power or battery in small size with high volume and lighter weight is welcomed, so that the sensor system can work for a long period. In addition, the ability of data processing onboard should be improved as well.

\section{CONCLUSIONS}

The application of PHM will improve the visibility of package box, and the condition of the equipment can be predicted, according to the environmental parameters. And it decreases the cost of logistics support, simplifies the training of technician. With the development of sensor technology, multi-sensor information fusion, intelligent inference technology, it will come true to estimate the health status of equipment, and diagnose the fault and locate it.

\section{REFERENCES}

[1] FENG Fu-zhou, SI Ai-wei, XING Wei, and et al, "Applications and Development of Malfunction Prognostics and Health Management Technologies," Journal of Academy of Armored Force Engineering, vol.23, Jun. 2009, pp.1-15.

[2] ZHANG Bao-zhen, and ZENG Tian-xiang, "PHM: The Key Enabler to F-35's Affordability," Aviation Maintenance \& Engineering, Jun. 2005, pp.20-23.

[3] Patrick W., Carl S., P.E., and et al, "Defining PHM, a LexicalEvolution of Maintenance and Logics," IEEE, Jun. 2006, pp.353-358.
[4] Chin Sun, Kevin Nguyen, Long Vu, and et al, "Prognostic/Diagnostic Health Management System (PHM) for Fab Efficiency,” IEEE, Jun. 2006, pp.433 438.

[5] Richard M., and Tonci Grubic, "Modeling Condition-Based Maintenance to Deliver a Service to Machine Tool Users," Int J Adv Manuf Technol, vol. 52, 2011, pp.1117-1132.

[6] ZENG Sheng-kui, Michael G. Pecht, and WU Ji, "Status and Perspectives of Prognostics and Health Management Technologies," Acta Aeronautica Et Astronautica Sinica, vol.26, May. 2005, pp.626-632.

[7] Zhang Bao-zhen, "Development and Applications of Integrated Diagnostics, Prognostics and Health Management Technologies of Abroad," Computer Measurement \& Control, vol.16, May. 2008, pp.591-594.

[8] Wu Ming-hui, Xu Ai-qiang, and Dai Hao-min, "Application of PHM Technic in Integrated Avionics Electronic System," Ordnance Industry Automation, vol.32, Apr. 2013, pp.72-77.

[9] LIU Jin-ning, ZHAO Jin-cheng, and WANG Chang-an, "Information Model Research of Prognostic and Health Management System," Instrumentation Technology, Mar. 2013, pp.8-11.

[10] WANG Han-zhong, YANG Jiang-ping, and WANG Shi-hua, "Research on the Maintenance Support System for Radar Equipment Based on the PHM," Journal of Academy of Armored Force Engineering, vol.19, Apr. 2008, pp.83-86.

[11] LIU Peng-peng, ZUO Hong-fu, and SUN Jian-zhong, "Study on Aircraft Maintenance Decision Theory in PH M System," Aeronautical Manufacturing Technology, vol. 20, 2012, pp.46-49. 